\title{
Performance of Multiway Relay DS-CDMA Systems over Nakagami- $m$ Fading Channels
}

\author{
Jia Shi and Lie-Liang Yang \\ School of ECS, University of Southampton, SO17 1BJ, United Kingdom \\ Tel: 0044-(0)23-8059 3364, Email: js22g09,1ly@ecs.soton.ac.uk, http://www-mobile.ecs.soton.ac.uk
}

\begin{abstract}
A multiway relay direct-sequence code-division multiple-access (MR-DS-CDMA) system is proposed for exchanging information among a group of distributed mobile terminals (MTs). In order to implement information exchange, a MT having similar distances from the other MTs is chosen to act as a relay, which assists the whole group of MTs to achieve their information exchange within two time-slots. In our proposed MR-DSCDMA system, the relay is operated in detection-and-forward (DF) relaying strategy. Signals at the relay and the MTs are detected in the principles of minimum mean-square error multiuser detection (MMSE-MUD) or of the receiver multiuser diversity assisted multi-stage MMSE MUD (RMD/MS-MMSE MUD). The error performance of the MR-DS-CDMA is investigated, when communicating over Nakagami- $m$ fading channels. Our studies show that the MR-DS-CDMA employing the RMD/MS-MMSE MUD at both the relay and MTs constitutes a high-efficiency information exchange system. It has low delay, promising error performance and low-complexity. Furthermore, it is robust to system overloading, where the number of MTs supported is higher than the spreading factor of the DS-CDMA system.
\end{abstract}

\section{INTRODUCTION}

In wireless communications, cooperative communications [1] have been becoming more and more attractive in recent years, in order to meet the requirements of the future generation wireless communication systems. In order to accomplish cooperation with high efficiency, a group of mobile terminals (MTs) in cooperation are required to share some of their information, which includes, such as, transmission power levels, channel state information (CSI), locations, transmitter processing vectors, etc. Additionally, there are some wireless networks, such as the wireless ad-hoc networks for video conference or multi-player gaming, which are designed motivating to share information among a group of distributed terminals. In these types of wireless systems where a group of terminals are required to share part or full of their information, one of the efficient communication strategies may be the multiway communication, which has drawn an increasing attention recently [2-6].

Multiway communication was investigated in [7] as early as in 1977, where the achievable rate region was studied. Recently, multi-way communication has been suggested to be implemented with the aid of relays, forming the multiway relay communication [2-6]. Specifically, in [2], the symmetric rate achievable by all users involved has been studied, when amplify-and-forward (AF), decode-and-forward (DF) or compress-and-forward $(\mathrm{CF})$ relaying protocol is invoked. In $[3,4]$, the multiway relay communication has been considered for a group of single-antenna MTs supported by a half-duplex multiantenna relay station, when non-regenerative [3] or regenerative [4] relaying strategy is considered. The capacity of the binary multiway relay channel has been studied in [5], where multiple terminals exchange their information with the aid of a relay supported by a so-called functional-decode-forward coding strategy. Furthermore, in [6], non-coherent fast frequencyhopping $(\mathrm{FFH})$ technique has been proposed for information exchange among a group of MTs through a relay, which recovers and forwards a time-frequency matrix. The information exchange in [6] is achieved using two time-slots regardless of the number of MTs involved.

In this contribution, we propose and investigate a multiway relay DS-CDMA (MR-DS-CDMA) system, which consists of a group of distributed MTs exchanging their information. During information exchange, one of the MTs preferably at the center of the MTs acts as the relay. In our MR-DS-CDMA system, information exchange is accomplished within two time-slots under the DF protocol, no matter how many MTs are involved. During the first time-slot, all MTs (except the relay itself) send their signals to the relay based on the DS-CDMA principles [10]. The relay extracts the other MTs' information from the DS-CDMA signals received from the first time-slot. Then, within the second time-slot, the relay broadcasts its own as well as the other MTs' information also based on the DS-CDMA principles. Finally, except the relay, any of the MTs is capable of obtaining the information of the other MTs through detection of the signals received from the relay within the second timeslot.

Due to multiuser interference (MUI) existing among the MTs, we can know that the achievable error performance of MR-DS-CDMA systems relies strictly on the detectors applied at both the relay and the MTs. In this contribution, two types of multiuser detectors (MUDs) having similar complexity are investigated in the context of the MR-DS-CDMA system. The first one is the conventional minimum mean-square error MUD (MMSE-MUD) [10,11], while the second one is the receiver multiuser diversity assisted multi-stage MMSE MUD (RMD/MS-MMSE MUD) [8,9], which has been found is capable of achieving near-optimum error performance. The error performance of the MR-DS-CDMA systems using various combinations of MMSE-MUD and RMD/MS-MMSE MUD is investigated, when assuming communications over Nakagami$m$ fading channels [12]. Our studies show that the MR-DSCDMA employing RMD/MS-MMSE MUD at both the relay and the MTs is a high-efficiency information exchange system. It has relatively low delay, requiring only two time-slots for information exchange among a group of MTs. It significantly outperforms the MR-DS-CDMA system employing the MMSEMUD at the relay or/and at the MTs, in terms of their achievable error performance. Furthermore, the RMD/MS-MMSE MUD is low-complexity, as the analysis in $[8,9]$ shows. Additionally, the MR-DS-CDMA employing RMD/MS-MMSE MUD at 
both the relay and the MTs is capable of supporting overloading and still achieving good error performance at reasonable signalto-noise ratio (SNR).

\section{SySTEM Models}

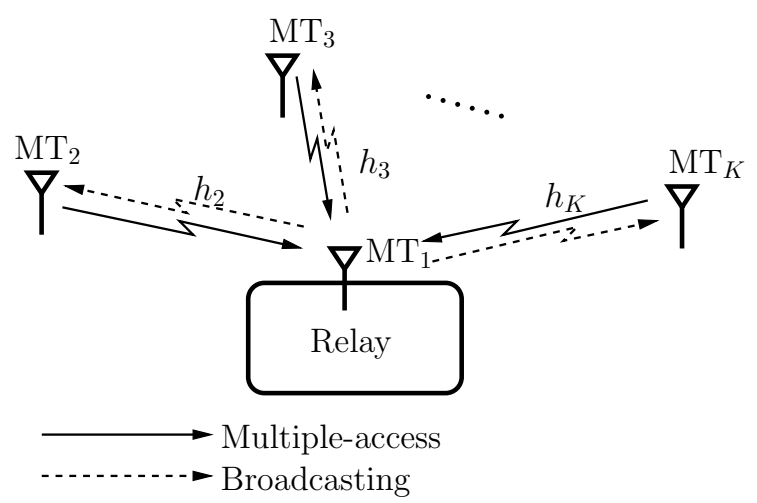

Fig. 1. Schematic of the DS-CDMA aided Multiway Relay system

The proposed MR-DS-CDMA system, as shown in Fig. 1, consists of $K$ MTs of equal priorities, each of which employs one antenna for receiving and transmission. The $K$ MTs are assumed to be close to each other. In order to carry out information exchange efficiently, one of the MTs is chosen based on certain rules to act as a (temporary) relay. For example, when distance-based rules are considered, the MT seating at a location that has short and similar distances from the other MTs is chosen as the relay. In our case, as shown in Fig. 1, we assume for simplicity that $\mathrm{MT}_{1}$ is chosen as the relay. In order to exchange the information among the $K \mathrm{MTs}$, the MRDS-CDMA system is operated in two modes: multiple-access (MA) and broadcasting (BC), supported by two time-slots for one period of information exchange. In the first time-slot, the $(K-1)$ MTs send their information to their relay of $\mathrm{MT}_{1}$ using the MA mode. At $\mathrm{MT}_{1}$, the information sent by the $(K-1)$ MTs can be detected based on certain detection schemes, as detailed in Section III. Up to this point, $\mathrm{MT}_{1}$ has obtained the information of all the $K$ MTs (including its own). During the second time-slot, $\mathrm{MT}_{1}$ conveys the information of all the $K$ MTs to each of the other MTs using the BC mode. Finally, at each of the $(K-1)$ MTs, the other MTs' information can be extracted based on certain detection schemes, as detailed in Section III.

In the considered MR-DS-CDMA system, we assume that the $K$ MTs are close to each other. This assumption is reasonable for some practical scenarios. For example, when a group of MTs motivate cooperation, the MTs close to each other are usually preferably chosen. Another example is that an adhoc network operated in one room consists of a number of MTs exchanging their information. Under the above assumption, we further assume that the MA/BC channels as seen in Fig. 1 experience independent identically distributed (iid) flat Nakagami- $m$ fading without considering the large-scale fading, including propagation pass-loss and shadowing. Furthermore, the MA and BC channels are reciprocal. Based on the above assumptions, it can be shown that, during the first time-slot, the DS-CDMA signal received by $\mathrm{MT}_{1}$ from the MA channels can be written as

$$
y=H x+n
$$

where, when assuming a spreading factor of $N, \boldsymbol{y}$ is an $N$ length observation vector, $\boldsymbol{x}=\left[x_{2}, x_{3}, \ldots, x_{K}\right]^{T}$ is an $(K-1)$ length vector containing the data symbols transmitted by the $(K-1)$ MTs. It is assumed that $E\left[\left|x_{k}\right|^{2}\right]=1$. In (1), $\boldsymbol{H}=$ $\boldsymbol{C A}$, where $\boldsymbol{C}=\left[\boldsymbol{c}_{2}, \boldsymbol{c}_{2}, \cdots, \boldsymbol{c}_{K}\right]$ is a spreading matrix with $\boldsymbol{c}_{k}, k=2, \ldots, K$, the spreading sequence assigned to $\mathrm{MT}_{k}$. We assume that $\left\{\boldsymbol{c}_{k}\right\}$ are random binary spreading sequences and $\boldsymbol{c}_{k}$ is normalized to satisfy $\left\|\boldsymbol{c}_{k}\right\|^{2}=1$. The matrix $\boldsymbol{A}$ is a diagonal matrix expressed as $\boldsymbol{A}=\operatorname{diag}\left\{a_{2}, a_{3}, \ldots, a_{K}\right\}$, where $a_{k}$ accounts for the fading of the $k$ th MA channel. Since we assume that the MA/BC channels experience Nakagami- $m$ fading, $\left|a_{k}\right|$ is hence obeys the Nakagami- $m$ distribution with the probability density function (PDF) [13]

$$
f_{\left|a_{k}\right|}(r)=\frac{2 m^{m} r^{2 m-1}}{\Gamma(m) \Omega^{m}} e^{-(m / \Omega) r^{2}}
$$

where $\Omega=E\left[\left|a_{k}\right|^{2}\right]$ and $m$ is the Nakagami- $m$ fading parameter characterizing the severity of fading. Finally, in (1), $n$ is an $N$-length Gaussian noise vector, which obeys the multivariate Gaussian distribution associated with zero mean and a covariance matrix $2 \sigma^{2} \boldsymbol{I}_{N}$, where $\sigma^{2}=1 /\left(2 \bar{\gamma}_{s}\right)$ with $\bar{\gamma}_{s}$ denoting the average signal-to-noise ratio (SNR) per symbol and $\boldsymbol{I}_{N}$ is an $(N \times N)$ identity matrix.

The relay $\mathrm{MT}_{1}$ detects for $x$ using certain detection algorithm, as detailed in Section III. Let after the detection the estimate to $x$ be expressed as $\hat{x}$. Then, the relay adds its own date $x_{1}$ to $\hat{x}$ to form $\tilde{\boldsymbol{x}}=\left[x_{1}, \hat{\boldsymbol{x}}^{T}\right]^{T}$. Finally, the relay transmits $\tilde{\boldsymbol{x}}$ with the aid of a random spreading matrix $\tilde{\boldsymbol{C}}=\left[\boldsymbol{c}_{1}, \boldsymbol{C}\right]$ over the BC channels. From the BC channels, $\mathrm{MT}_{k}$ can obtain the observations expressed in matrix form as

$$
\tilde{\boldsymbol{y}}_{k}=\tilde{\boldsymbol{H}}_{k} \tilde{\boldsymbol{x}}+\tilde{\boldsymbol{n}}_{k}, k=2, \ldots, K
$$

where $\boldsymbol{y}_{k}, \tilde{\boldsymbol{H}}_{k}, \tilde{\boldsymbol{x}}$ and $\tilde{\boldsymbol{n}}_{k}$ have similar explanations as those in (1), except that $\tilde{\boldsymbol{x}}$ is $K$-length and $\tilde{\boldsymbol{H}}_{k}=a_{k} \tilde{\boldsymbol{C}}$ with $a_{k}$ being the channel gain of the $k$ th $\mathrm{BC}$ channel.

Finally, at $\mathrm{MT}_{k}, k=2, \ldots, K, \tilde{x}$ is detected according to certain detection algorithm, in order to recover the information transmitted by the MTs other than $\mathrm{MT}_{k}$. Let us now consider the detection strategies used by the relay and other MTs.

\section{Detection Algorithms}

In this paper, two detection algorithms are considered for the MR-DS-CDMA systems. The first one is the well-known MMSE-MUD, while the second one is the RMD/MS-MMSE MUD proposed and investigated in [8,9]. Either of them may be employed by the relay or by the MTs. Note that, since the MIMO equation (3) has the same form as (1), we hence describe the detection algorithms only according to (1). The detection algorithms based on (3) can be obtained directly by replacing the corresponding terms.

\section{A. MMSE-MUD}

It is well-known that, when the MMSE-MUD is employed, the decision variables for $x$ in (1) is given by [10]

$$
\boldsymbol{z}=\boldsymbol{W}^{H} \boldsymbol{y}, \text { or } z_{k}=\boldsymbol{w}_{k}^{H} \boldsymbol{y}, k=2, \ldots, K
$$


decision variable vector, $\boldsymbol{W}=\left[\boldsymbol{w}_{2}, \ldots, \boldsymbol{w}_{K}\right]$ is an $(N \times(K-$ $1)$ ) weight matrix, while $\boldsymbol{w}_{k}$ is an $N$-length weight vector, $W$ and $\boldsymbol{w}_{k}$ are expressed as [10],

$$
\boldsymbol{W}=\boldsymbol{R}_{y}^{-1} \boldsymbol{H}, \quad \boldsymbol{w}_{k}=\frac{\boldsymbol{R}_{k}^{-1} \boldsymbol{h}_{k}}{1+\boldsymbol{h}_{k}^{H} \boldsymbol{R}_{k}^{-1} \boldsymbol{h}_{k}}
$$

where $\boldsymbol{R}_{y}$ and $\boldsymbol{R}_{k}$ denote, respectively, the autocorrelation matrix of $\boldsymbol{y}$ and the autocorrelation matrix of the interferenceplus-noise, which are

$$
\begin{aligned}
& \boldsymbol{R}_{y}=\boldsymbol{H} \boldsymbol{H}^{H}+2 \sigma^{2} \boldsymbol{I}_{N}=\sum_{l=2}^{K} \boldsymbol{h}_{l} \boldsymbol{h}_{l}^{H}+2 \sigma^{2} \boldsymbol{I}_{N} \\
& \boldsymbol{R}_{k}=\sum_{l \neq k}^{K} \boldsymbol{h}_{l} \boldsymbol{h}_{l}^{H}+2 \sigma^{2} \boldsymbol{I}_{N}=\boldsymbol{R}_{y}-\boldsymbol{h}_{k} \boldsymbol{h}_{k}^{H}
\end{aligned}
$$

When applying the Gaussian approximation on the multiuser interference after the MMSE-MUD, it can be shown that the inphase component $z_{k}^{(I)}$ and quadrature component $z_{k}^{(Q)}$ of $z_{k}$ obey the PDF

$$
f\left(z_{k}^{(\cdot)} \mid x_{k}^{(\cdot)}\right)=\frac{1}{\sqrt{2 \pi} \sigma_{k}^{(\cdot)}} \exp \left[-\frac{\left(z_{k}^{(\cdot)}-m_{k}^{(\cdot)}\right)^{2}}{2 \sigma_{k}^{(\cdot) 2}}\right]
$$

where $z_{k}^{(\cdot)}$ denotes $z_{k}^{(I)}$ or $z_{k}^{(Q)}$ and $x_{k}^{(\cdot)}$ denotes $x_{k}^{(I)}$ or $x_{k}^{(Q)}$, the mean and variance in the above equation are given by

$$
m_{k}^{(\cdot)}=\frac{\bar{\gamma}_{k}}{1+\bar{\gamma}_{k}} x_{k}^{(\cdot)}, \quad \sigma_{k}^{(\cdot) 2}=\frac{\bar{\gamma}_{k}}{2\left(1+\bar{\gamma}_{k}\right)^{2}}
$$

where $\bar{\gamma}_{k}=\boldsymbol{h}_{k}^{H} \boldsymbol{R}_{k}^{-1} \boldsymbol{h}_{k}$ represents the instantaneous signal-tointerference-plus-noise ratio (SINR) for detection of user $k$.

Additionally, as discussed in [9], when the RMD/MS-MMSE MUD is applied for the multiuser systems with I-Q type baseband modulation, such as $M$-ary quadrature amplitude modulation (MQAM), it is desirable that the real and imaginary parts of a transmitted symbol are separately detected based on an equivalent real-domain MIMO equation formed from (1). This equivalent real-domain MIMO equation can be expressed as

$$
\boldsymbol{y}_{R}=\boldsymbol{H}_{R} \boldsymbol{x}_{R}+\boldsymbol{n}_{R}
$$

where

$$
\begin{aligned}
\boldsymbol{y}_{R} & =\left[\Re\left\{\boldsymbol{y}^{T}\right\}, \Im\left\{\boldsymbol{y}^{T}\right\}\right]^{T}, \boldsymbol{x}_{R}=\left[\left(\boldsymbol{x}^{(I)}\right)^{T},\left(\boldsymbol{x}^{(Q)}\right)^{T}\right]^{T} \\
\boldsymbol{H}_{R} & =\left[\begin{array}{cc}
\Re\{\boldsymbol{H}\} & -\Im\{\boldsymbol{H}\} \\
\Im\{\boldsymbol{H}\} & \Re\{\boldsymbol{H}\}
\end{array}\right], \boldsymbol{n}_{R}=\left[\begin{array}{l}
\Re\{\boldsymbol{n}\} \\
\Im\{\boldsymbol{n}\}
\end{array}\right]
\end{aligned}
$$

Then, when the MMSE-MUD is applied based on (10), the decision variable vector $z$ for the $(K-1)$ users or, specifically, the decision variable $z_{k}^{(I)}$ (or $z_{k}^{(Q)}$ ) for $x_{k}^{(I)}\left(x_{k}^{(Q)}\right)$ can be expressed as

$$
\boldsymbol{z}=\boldsymbol{W}^{T} \boldsymbol{y}_{R}, z_{k}^{(\cdot)}=\boldsymbol{w}_{k}^{(\cdot) T} \boldsymbol{y}_{R}, k=2, \ldots, K
$$

where $\boldsymbol{W}$ and $\boldsymbol{w}_{k}^{(\cdot)}$ optimized in MMSE sense are given by

$$
\begin{aligned}
\boldsymbol{W} & =\left(\boldsymbol{H}_{R} \boldsymbol{H}_{R}^{T}+2 \sigma^{2} \boldsymbol{I}_{2 N}\right)^{-1} \boldsymbol{H}_{R}, \\
\boldsymbol{w}_{k}^{(\cdot)} & =\frac{\boldsymbol{R}_{k}^{-1} \boldsymbol{h}_{k}^{(\cdot)}}{1+\boldsymbol{h}_{k}^{(\cdot) T} \boldsymbol{R}_{k}^{-1} \boldsymbol{h}_{k}^{(\cdot)}}, k=1,2, \ldots, K
\end{aligned}
$$

where $\boldsymbol{R}_{k}$ is the autocorrelation matrix of interference-plusnoise expressed as $\boldsymbol{R}_{k}=\boldsymbol{H}_{R} \boldsymbol{H}_{R}^{T}+2 \sigma^{2} \boldsymbol{I}_{2 N}-\boldsymbol{h}_{k}^{(\cdot)} \boldsymbol{h}_{k}^{(\cdot) T}$. Furthermore, as shown in [9], $z_{k}^{(\cdot)}$ in (12) has the PDF of (8) associated with the SINR of $\bar{\gamma}_{k}^{(\cdot)}=\boldsymbol{h}_{k}^{(\cdot) T} \boldsymbol{R}_{k}^{-1} \boldsymbol{h}_{k}^{(\cdot)}$.

\section{B. RMD/MS-MMSE MUD}

The RMD/MS-MMSE MUD [8,9] is build on the MMSEMUD analyzed in the above section. It is operated in the principles successive interference cancellation (SIC), as detailed in $[8,9]$. The RMD/MS-MMSE MUD can be briefly described as the following steps:

Step 1: Initialization;

Step2: for $n=1,2, \ldots,(K-1)$ if one-dimensional modulation scheme is employed, or $n=1,2, \ldots, 2(K-1)$ if two-dimensional modulation scheme is employed, executing the following operations:

1) MMSE-MUD;

2) Finding the most reliable among the symbols having not been detected according to their reliabilities measured based on a scheme as shown below;

3) Making a decision for the most reliable symbol;

4) Canceling the component due to the detected symbol from the observation equation (1) or (10);

5) Updating the weight matrix (vector) used by the MMSEMUD;

6) Setting $n=n+1$ and returning to 1 ) until all the symbols are detected.

In the above algorithm for the RMD/MS-MMSE MUD, when binary phase-shift keying (BPSK) is employed and assume that the source data bits obey independent identical distribution (iid), the reliabilities measured in maximum a-posterior (MAP) principles is given by

$$
\begin{aligned}
L_{k} & =\left|\ln \left[\frac{f\left(x_{k}=+1 \mid z_{k}^{(I)}\right)}{f\left(x_{k}=-1 \mid z_{k}^{(I)}\right)}\right]\right| \\
& =\left|\ln \left[\frac{f\left(z_{k}^{(I)} \mid x_{k}=+1\right)}{f\left(z_{k}^{(I)} \mid x_{k}=-1\right)}\right]\right|, k=2, \ldots, K
\end{aligned}
$$

Upon applying the PDF of (8) associated with $x_{k}^{(I)}=+1$ or -1 into above equation, we obtain [8]

$$
L_{k}=\left(1+\bar{\gamma}_{k}\right)\left|z_{k}^{(I)}\right|, k=2,3, \ldots, K
$$

By contrast, when the I-Q modulation, such as MQAM, is employed, given the decision variables $z_{k}^{(I)}\left(z_{k}^{(Q)}\right)$ as shown in (12) for $x_{k}^{(I)}\left(x_{k}^{(Q)}\right)$, where $x_{k}^{(I)}\left(x_{k}^{(Q)}\right) \in \mathcal{S}^{\prime}=$ $\left\{s_{0}, \ldots, s \sqrt{M}-1\right\}$, according to [9], the reliabilities of $x_{k}^{(I)}$ $\left(x_{k}^{(Q)}\right)$ can be evaluated in MAP sense by the formula

$$
\begin{gathered}
L_{k}^{(\cdot)}=\frac{\max _{s_{i} \in \mathcal{S}^{\prime}}\left\{\exp \left[-\left(\frac{1+\bar{\gamma}_{k}^{(\cdot)}}{\sqrt{\bar{\gamma}_{k}^{(\cdot)}}} z_{k}^{(\cdot)}-\sqrt{\bar{\gamma}_{k}^{(\cdot)}} s_{i}\right)^{2}\right]\right\}}{\sum_{s_{j} \in \mathcal{S}^{\prime}} \exp \left[-\left(\frac{1+\bar{\gamma}_{k}^{(\cdot)}}{\sqrt{\bar{\gamma}_{k}^{(\cdot)}}} z_{k}^{(\cdot)}-\sqrt{\bar{\gamma}_{k}^{(\cdot)} y} s_{j}\right)^{2}\right]}, \\
k=2, \ldots, 2 K-1
\end{gathered}
$$




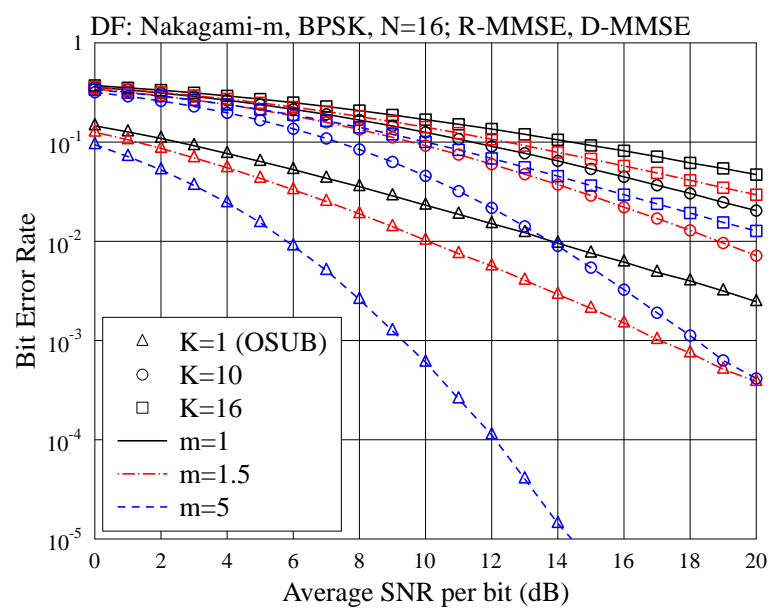

Fig. 2. BER performance of MR-DS-CDMA systems where both relay and MTs use MMSE-MUD, when communicating over Nakagami- $m$ fading channels.

Additionally, in the RMD/MS-MMSE MUD algorithm, given the initial weight matrix $\boldsymbol{W}^{(0)}$, the weight matrix at the following iterations can be updated according to the formula [9]

$$
\boldsymbol{W}^{(s)}=\left[\boldsymbol{W}^{(s-1)}+\frac{\boldsymbol{w}_{k_{I}^{(s-1)}} \boldsymbol{h}_{k_{I}^{(s-1)}}^{(I) T} \boldsymbol{W}^{(s-1)}}{1-\boldsymbol{h}_{k_{I}^{(s-1)}}^{(I) T} \boldsymbol{w}_{k_{I}^{(s-1)}}}\right] \boldsymbol{P}^{(s)},
$$

if the real part of a symbol is detected, or

$$
\boldsymbol{W}^{(s)}=\left[\boldsymbol{W}^{(s-1)}+\frac{\boldsymbol{w}_{k_{Q}^{(s-1)}} \boldsymbol{h}_{k_{Q}^{(Q)-1)}}^{(Q) T} \boldsymbol{W}^{(s-1)}}{1-\boldsymbol{h}_{k_{Q}^{(s-1)}}^{(Q) T} \boldsymbol{w}_{k_{I}^{(s-1)}}}\right] \boldsymbol{P}^{(s)} .
$$

when the imaginary part of a symbol is detected. In (17) and (18), $\boldsymbol{w}_{k_{I}^{(s-1)}}, \boldsymbol{w}_{k_{Q}^{(s-1)}}, \boldsymbol{h}_{k_{I}^{(s-1)}}^{(I)}, \boldsymbol{h}_{k_{Q}^{(s-1)}}^{(Q)}$ correspond to the symbol detected at the $(s-1)$ th detection stage, $\boldsymbol{P}^{(s)}$ is a permutation matrix obtained from $I_{2 N}$ by deleting the columns corresponding to the symbols having been detected.

\section{Performance Results}

Figs. 2 - 4 show the BER performance of the MR-DS-CDMA systems using BPSK modulation, when different combinations of detection algorithms at relay and MTs are employed. Specifically, in the context of Fig. 2, the conventional MMSE-MUD is employed at both the relay and the MTs. For Fig. 3, the relay employs the RMD/MS-MMSE MUD, while the MTs employ the conventional MMSE-MUD. Finally, in the MR-DS-CDMA systems for Fig. 4, the RMD/MS-MMSE MUD is employed at both the relay and the MTs. In these figures, the curves notified as $K=1$ (OSUB) are the one-hop single-user bounds, which are the error probabilities of corresponding DS-CDMA systems supporting single user. Furthermore, in Figs. 3 and 4 when the RMD/MS-MMSE MUD is employed, the BER of overload MR-DS-CDMA systems supporting $K=25>16$ MTs was also depicted.

From the results of Figs. 2 - 4, we can observe that, when the channel fading becomes less severe, i.e., as the value of the fading parameter $m$ increases, the BER performance of the

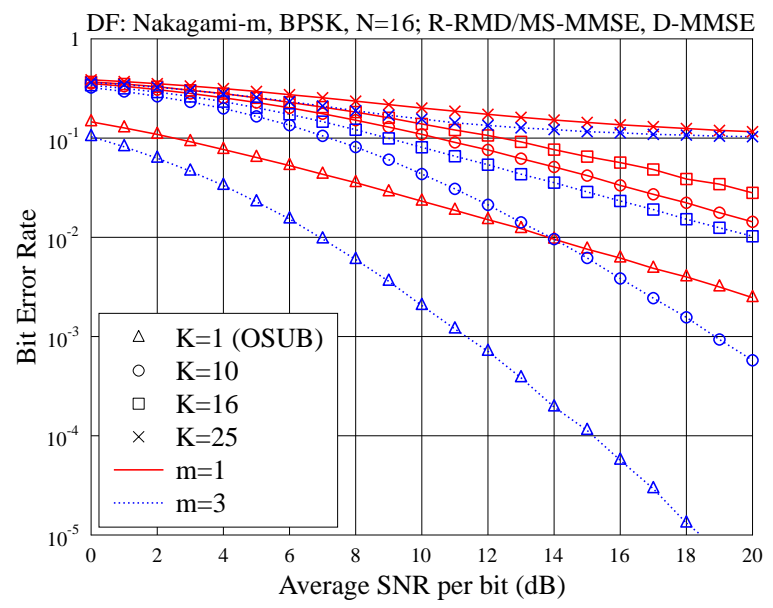

Fig. 3. BER performance of MR-DS-CDMA systems employing RMD/MSMMSE MUD at relay and MMSE-MUD at MTs, when communicating over Nakagami- $m$ fading channels.

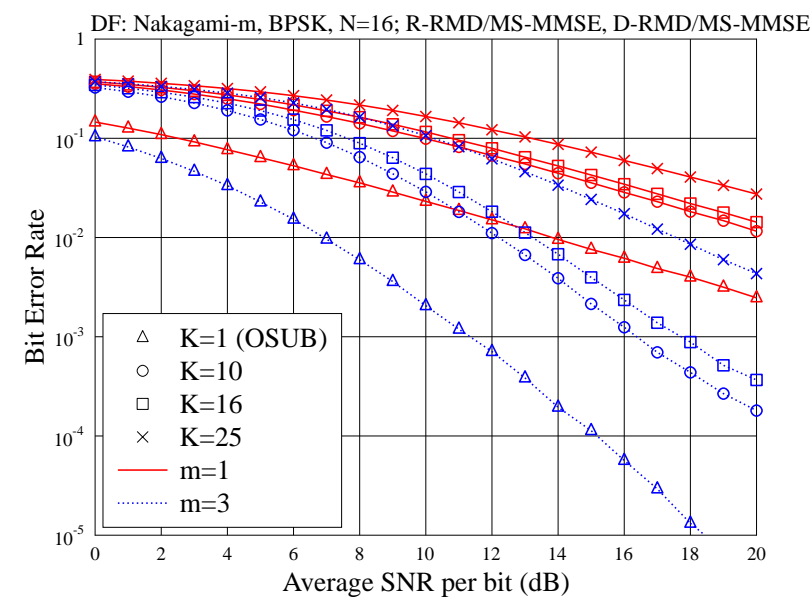

Fig. 4. BER performance of MR-DS-CDMA systems with both relay and MTs using RMD/MS-MMSE MUD, when communicating over Nakagami- $m$ fading channels.

MR-DS-CDMA systems improves. We can deduce from the results that the RMD/MS-MMSE MUD significantly outperforms the MMSE-MUD. This can be reflected by the following observations. First, when comparing the BER performance corresponding to the cases with $m=1$ and $K=16$, we can see that the BER performance in Fig. 3 is better than that in Fig. 2, while the BER performance in Fig. 4 is better than that in both Fig. 2 and Fig. 3. Second, when comparing Fig. 3 with Fig. 4, which considered similar cases, we can observe that, for the $K>1$ cases, the BER performance shown in Fig. 4 is usually much better than the corresponding BER performance shown in Fig. 3. Furthermore, when the RMD/MS-MMSE MUD is applied at both the relay and the MTs, as shown in Fig. 4, the MRDS-CDMA system is capable of achieving a reasonable BER performance, even though the system is significantly overload with $K=25>16$. Additionally, from the above observations, we can be implied that, in Fig. 3 when the RMD/MS-MMSE MUD is applied at the relay, the BER performance of the RMDDS-CDMA systems is mainly limited by that achieved by the 


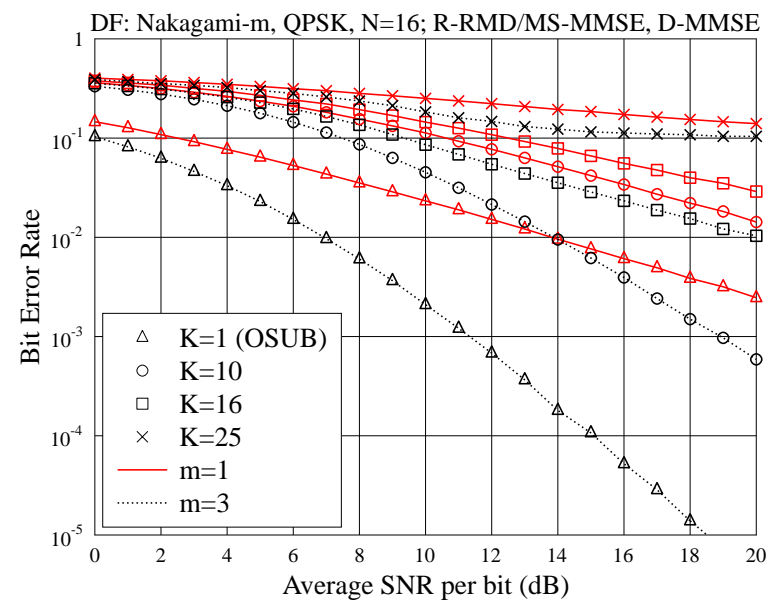

Fig. 5. BER performance of MR-DS-CDMA systems employing RMD/MSMMSE MUD at relay and MMSE-MUD at MTs, when communicating over Nakagami- $m$ fading channels.

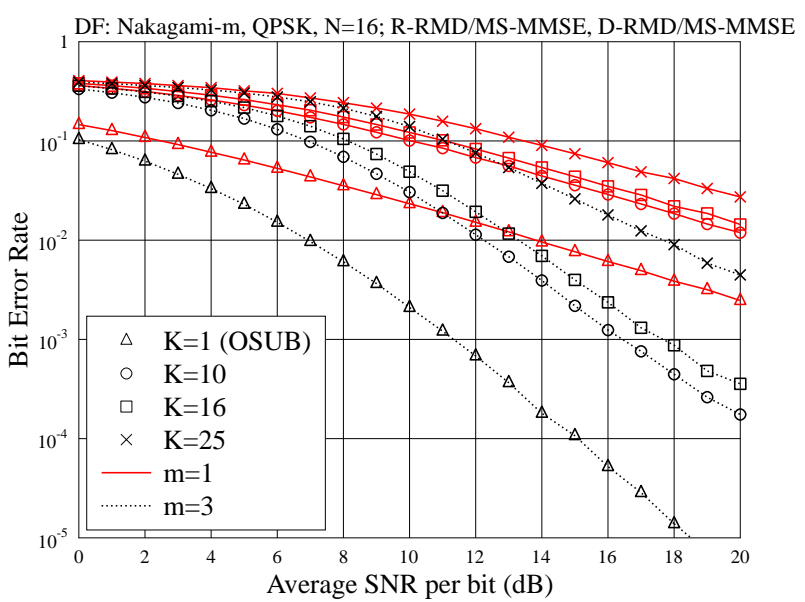

Fig. 6. BER performance of MR-DS-CDMA systems with both relay and MTs employing RMD/MS-MMSE MUD, when communicating over Nakagami- $m$ fading channels.

MMSE-MUD operated at the MTs.

Fig. 5 illustrates the BER performance of the MR-DSCDMA systems using QPSK modulation, when the MMSEMUD is employed at the MTs and the RMD/MS-MMSE MUD is applied at the relay. By contrast, Fig. 6 depicts the BER performance of the MR-DS-CDMA systems using QPSK modulation, when both the relay and MTs employ the RMD/MSMMSE MUD. The other parameters used for these two figures are similar, as shown in the figures. Furthermore, except the QPSK modulation for Figs. 5 and 6, the other settings for these two figures are the same as those in Figs. 3 and 4, where BPSK modulation is assumed. From Figs. 5 and 6 we can have similar observations as those from Figs. 3 and 4. As seen in Figs. 5 and 6, given the same number of MTs supported and the same fading parameter $m$, the BER performance shown in Fig. 6 is much better than that shown in Fig. 5, which implies that the RMD/MS-MMSE MUD significantly outperforms the conventional MMSE-MUD, especially, when the MR-DS-CDMA systems support a relatively high number of MTs.
Finally, we note that, in addition to that the RMD/MSMMSE MUD outperforms significantly the conventional MMSE-MUD in terms of their achievable error performance, the RMD/MS-MMSE MUD also has a low complexity, which only linearly increases with the number of MTs involved [8].

\section{Conclusions}

In this contribution, we have proposed and studied a multiway relay scheme based on DS-CDMA signaling, i.e., the MRDS-CDMA system. In our MR-DS-CDMA system, information exchange among MTs is always achieved within two time-slots, regardless of the number of MTs supported. The relay used in the MR-DS-CDMA system is operated in DF mode. Furthermore, two types of detection schemes, namely the MMSEMUD and RMD/MS-MMSE MUD, have been proposed for signal detection at the relay and at the MTs. Our studies and simulation results show that the MR-DS-CDMA system employing the RMD/MS-MMSE MUD at both the relay and the MTs constitutes one of the highly promising multiway relay schemes. The MR-DS-CDMA system is low delay, which requires only two time-slots for information exchange regardless of the number of MTs supported. It is capable of achieving near-optimum error performance as demonstrated in $[8,9]$ as well as in this paper. Furthermore, the RMD/MS-MMSE MUD invoked is low-complexity, which is only linearly dependent on the number of MTs involved.

\section{REFERENCES}

[1] S. Berger, M. Kuhn, A. Wittneben, T. Unger, and A. Klein, "Recent advances in amplify-and-forward two-hop relaying," IEEE Commun. Mag., vol. 47, no. 7, pp. 50-56, July 2009.

[2] D.Gunduz, A. Yener, A. Goldsmith, and H. Poor, "The multi-way relay channel," IEEE Inter. Sym. on Infor. Theory, pp. 339-343, July 2009.

[3] A.U.T. Amah and A. Klein, "Non-regenerative multi-way relaying with linear beamforming," in Proc. IEEE PIMRC'2009, Tokyo, Japan, 13-16 Sept. 2009.

[4] A.U.T. Amah and A. Klein, "A transceiver strategy for regenerative multiantenna multi-way relaying," in Proc. of the 3rd IEEE International Workshop on Computational Advances in multi-sensor adaptive processing, Aruba, 13 - 16 Dec. 2009

[5] L. Ong, S.J. Johnson and C.M. Kellett, "An optimal coding strategy for the binary multi-way relay channel," IEEE Communications Letters, Vol. 14, No. 4, pp. 330 - 332, April 2010.

[6] J.Cao, L.-L. Yang and Z. Zhong, "Noncoherent Multi-Way Relay Based on Fast Frequency-Hopping M-ary Frequency-Shift Keying," The Seventh International Symposium on Wireless Communications Systems (ISWCS), York, UK, Sept. 2010.

[7] E.C. van der Meulen, "A survey of multi-way channels in information theory:1961-1976," IEEE Trans. on Infor. Theory, vol. 23, no. 1, pp. 137, Jan 1977.

[8] L.-L. Yang, "Receiver Multiuser Diversity Aided Multi-Stage MMSE Multiuser Detection: A Low-Complexity Detector Fast-Converging to the Optimum," IEEE 71th Vehicular Technology Conference: VTC2010Spring, May 2010.

[9] L.-L. Yang, "Receiver Multiuser Diversity Aided Multi-Stage MMSE Multiuser Detection for DS-CDMA and SDMA Systems Employing I-Q Modulation," IEEE 72th Vehicular Technology Conference: VTC2010-Fall, September 2010.

[10] L.-L. Yang, Multicarrier Communications. Chichester, United Kingdom: John Wiley, 2009.

[11] S. Verdu, Multiuser Detection. C Cambridge University Press, 1998.

[12] M. Nakagami, "Statistical Methods in Radio Wave Propagation," W.C. Hoffman, Ed, Oxford, U.K, 1960.

[13] L.-L. Yang and L. Hanzo, "Performance of generalized multicarrier DSCDMA over Nakagami-m fading channels," IEEE Trans. on Commun., vol. 50 , pp. 956 - 966, June 2002. 